\title{
Analysis on Current Limiting and Bus-voltage Sag Suppressing Characteristics of a Flux-lock type SFCL using an Isolated Transformer
}

\author{
Shin-Won Lee \\ Department of Computer Engineering, Jungwon University, Chungbuk, 367-805, Korea. \\ ORCID: 0000-0002-4535-2996.
}

\begin{abstract}
In this paper, the current limiting and bus-voltage sag suppressing characteristics of a flux-lock type superconducting fault current limiter (SFCL) with an isolated transformer were analyzed through short-circuit tests for a simulated power system with the SFCL. The isolated transformer, which was connected to the superconducting (SC) element of the flux-lock type SFCL, was designed to improve the current limiting and the bus-voltage sag suppressing characteristics of the flux-lock type SFCL by preventing the fault current from directly passing through the SC element. An analysis of the experimental results considering the winding ratio of the isolated transformer confirmed that the flux-lock type SFCL, which was planned with a lower winding ratio, performed the limiting operation more effectively, while the flux-lock type SFCL with a higher winding ratio of the isolated transformer, was more effective from the point of view of the suppression of the bus-voltage sag.
\end{abstract}

Keywords: fault current, flux-lock type SFCL, bus-voltage sag, isolated transformer

\section{INTRODUCTION}

Due to year-to-year increasing power demands, the complexity of power systems and the reduction of power impedance exceed the volume of short circuits of existing breakers for a fault current occurring from a short circuit and will seriously cause stability problems such as thermal shock and mechanical shock [1-3]. Many papers have proposed the replacement of large breakers or power equipment of high impedance, installation of serial reactors, and separation of linkage lines as an alternative plan, but economic problems and technical stability of power still remain to be solved [4-6].

The ideal alternative plan to more effectively control the fault current is to use the superconducting current limiter (SFCL), using superconducting properties [7-9]. It actively advances the development of the SFCL and applications for practical use. Various types of SFCL have now been developed [10-14]. The flux-lock type SFCL has an advantage in reducing the burden of limiter impedance and the SC element to control the winding direction and inductance, while it has a disadvantage in decreasing the fault current limiting effect that is due to conduction of a partial fault current through a direct device, in that after removing the fault current, saturation of the iron core occurs causing a longer recovery time [15-17].

In this study, in the occurrence of a fault current, we configured the system to connect an isolated transformer between superconducting elements to configure the flux-lock type SFCL. In this way we avoid conduction of a direct fault current through a superconducting element. For our analysis, we simulated the current limiting characteristics of the flux-lock type SFCL, which included an isolated transformer. When a fault occurred, we measured the quench time according to changes in the secondary coil winding ratio of the isolated transformer and the characteristics of the bus-voltage sag suppressing operation. After the fault was removed, we analyzed the recovery characteristics and consumption power with the existing magnetic flux SFCL.

\section{EXPERIMENTAL METHODES}

The composition of the flux-lock type SFCL is two coils connected in parallel or serially via one ferromagnetic iron core, and the superconducting element is connected to one of the two coils. In this experiment, as shown in Figure 1, this test device was constructed with the two coils connected in parallel and the isolated transformer between the superconducting elements was connected serially to one of the coils. This system performed the simulated short circuit experiment for the characterization of the fault current limiting of the flux-lock type SFCL using an isolated transformer.

Figure 1 gives the structure of SFCL using a magnetic conjunction of two coils connected parallel, and the winding ratio of the two connected coils was 4:1. It was made to connect parallel via an iron core, and the winding ratio of the isolated transformer coils changed from 0.25 to 0.5 . The element of the SFCL used a high superconductor film of $\mathrm{YBa}_{2} \mathrm{Cu}_{3} \mathrm{O}_{7}(\mathrm{YBCO})$. To simulate the short circuit, the power supply voltage $\left[\mathrm{E}_{\mathrm{s}}\right]$ was applied as $120\left[\mathrm{~V}_{\mathrm{rms}}\right]$.

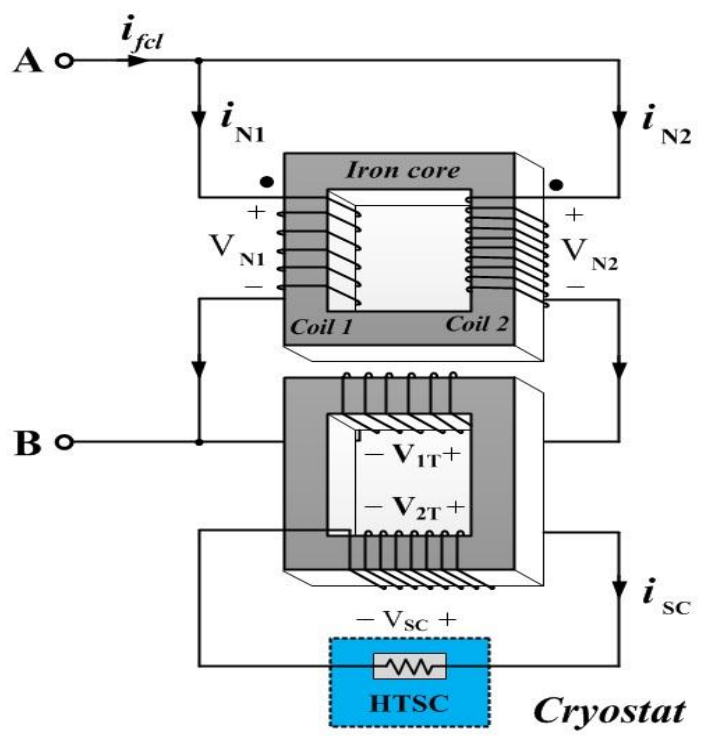

Figure 1. Structure of a flux-lock type SFCL using an isolated transformer 
Figure 2 shows the experimental circuit with a flux-lock type SFCL using an isolated transformer. $E_{S}$ and $\mathrm{R}_{S}$ represent the voltage and the resistance of the power source. $L_{1}, L_{2}, R_{1}$ and $R_{2}$ represent the inductance and the resistance of lines 1 and 2 , respectively. $\mathrm{R}_{\mathrm{L} 1}$ and $\mathrm{R}_{\mathrm{L} 2}$ represent the resistance of the load connected to each line. $i_{\mathrm{S}}, i_{1}$ and $i_{2}$ indicate the current of the power source and each line, respectively. The current is distributed according to the impedance of the lines because the total current is connected in parallel with the $i_{1}$ current and $i_{2}$ current. If a fault occurs in line 1 , then the $i_{1}$ current increases rapidly because the impedance decreases. The current $i_{2}$ on line 2 decreases according to the impedance changes. We expected that the current $i_{1}$ would change the magnitude of the current depending on the winding ratio and the impedance of the SFCL. After turning on the switch $\mathrm{SW}_{1}$, the switch $\mathrm{SW}_{2}$ was turned on for 5 cycles. With this experimental circuit, we analyzed the current limiting characteristics of the flux-lock type SFCL and the bus-voltage sag suppressing effect in the power system applied by the SFCL. In addition, we described the dependence of the current limiting and bus-voltage sag suppressing characteristics on the winding ratio of an isolated transformer and the winding direction of the two coils comprising the fluxlock type SFCL.

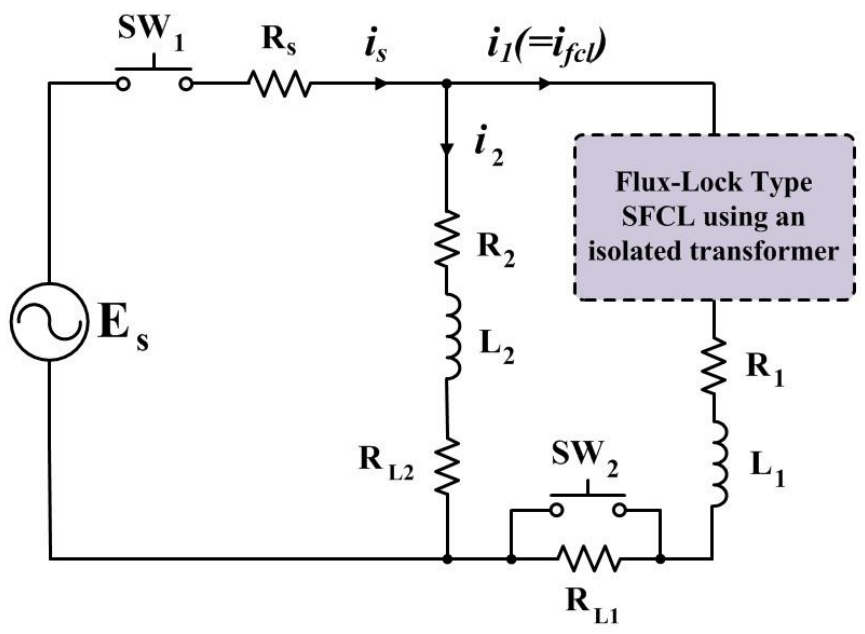

Figure 2. Experimental circuit with a flux-lock type SFCL using an isolated transformer

\section{RESULTS AND DISCUSSION}

Figure 3 shows the installation existence or absence of the isolated transformer and presents the characteristic waveform of the current and voltage when the winding ratio of the secondary coil $\left(n=N_{2 T} / N_{1 T}\right)$ was changed from $n=0.25$ to $n=0.5$. In Figure $3, i_{\mathrm{sc}}{ }^{0.25}$ and $i_{\mathrm{sc}}{ }^{0.5}$ are the currents of the superconducting element when the winding ratio of the isolated transformer is 0.25 and 0.5 , respectively. $i_{\mathrm{sc}}{ }^{\text {w/o }}$ is the current of the superconducting element when the isolated transformer is not installed. $v_{\mathrm{sc}}^{0.25}$ and $v_{\mathrm{sc}}^{0.5}$ are the voltages of the superconducting element, which correspond to the case in which the winding ratio of the isolated transformer is 0.25 and 0.5 , respectively. $v_{\mathrm{sc}}{ }^{\mathrm{w} / \mathrm{o}}$ is the voltage across the superconducting element in the flux-lock type SFCL without the isolated transformer.
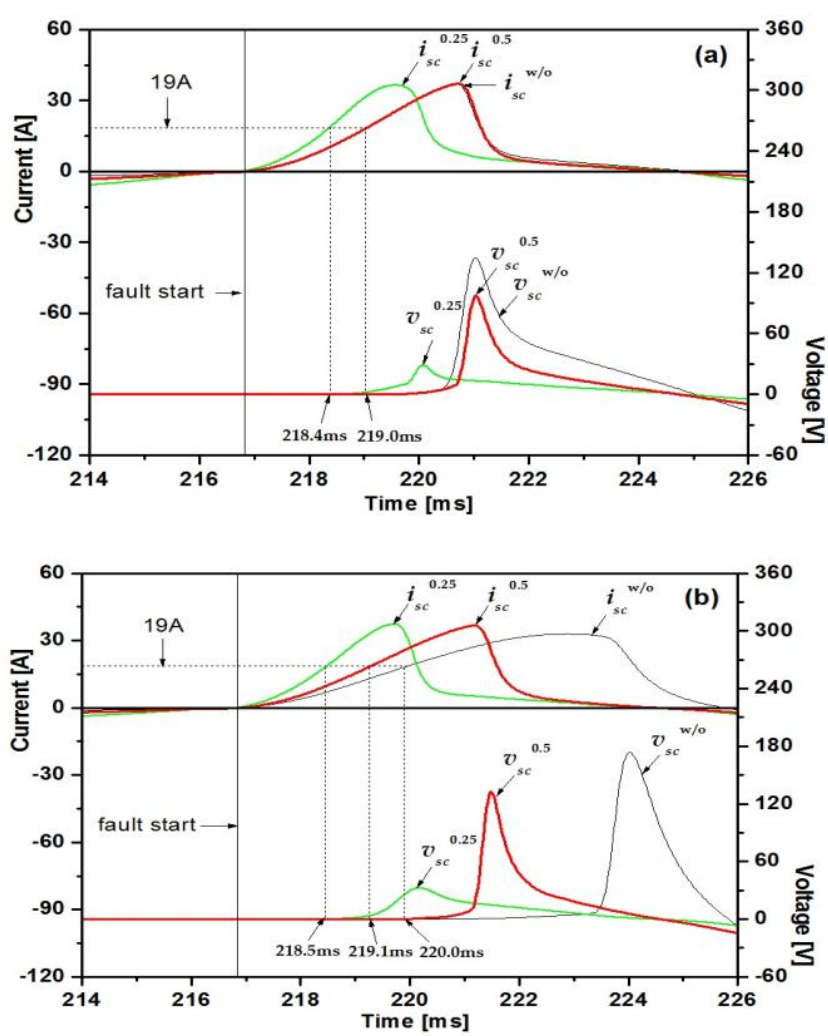

Figure 3. The waveform of the current and voltage of the fluxlock type SFCL according to the change of the winding ratio of an isolated transformer; (a) The additive polarity winding (b) The subtractive polarity winding

Figure 3(a) presents the additive polarity winding and shows the waveform of the voltage and current of the superconducting element when a fault occurs. The response time is the measured time of the voltage of the superconducting element when the critical current of this element exceeds 19A. The response times from the start of the fault to the occurring element voltage when there is no isolated transformer and the winding ratio being $\mathrm{n}=0.5$ are similar, 2.1 [ms], and in the case of the winding ratio being $\mathrm{n}=0.25,1.5[\mathrm{~ms}]$. When a fault occurs, the secondary coil winding ratio becomes smaller, and the superconducting element voltage becomes larger, and in the case of no isolated transformer, the element voltage is high.

Figure 3(b) gives the subtractive polarity winding and shows the waveform of the voltage and the current of the superconducting element when a fault occurs. When an isolated transformer is connected, the current of the superconducting element is similar to the additive polarity winding. When there is no isolated transformer, the peak current is late. The response time that is from the fault start timing $(216.9 \mathrm{~ms})$ to the occurring voltage of the superconducting element shows a similar reaction in comparison to the additive polarity winding. This element voltage shows the same trend as the additive polarity winding. The response time when there is no isolated transformer is 3.1 [ms]. The element voltage is higher in the case of the winding of the subtractive polarity winding than with the additive polarity winding because of the increase and decrease of the flux linkage occurring in an iron core as the winding of primary coil and secondary coil. This occurs when voltage is applied to the two coils. When a fault current occurs 
when an isolated transformer is used because the voltage is small, this system reduces the burden of the superconducting element.
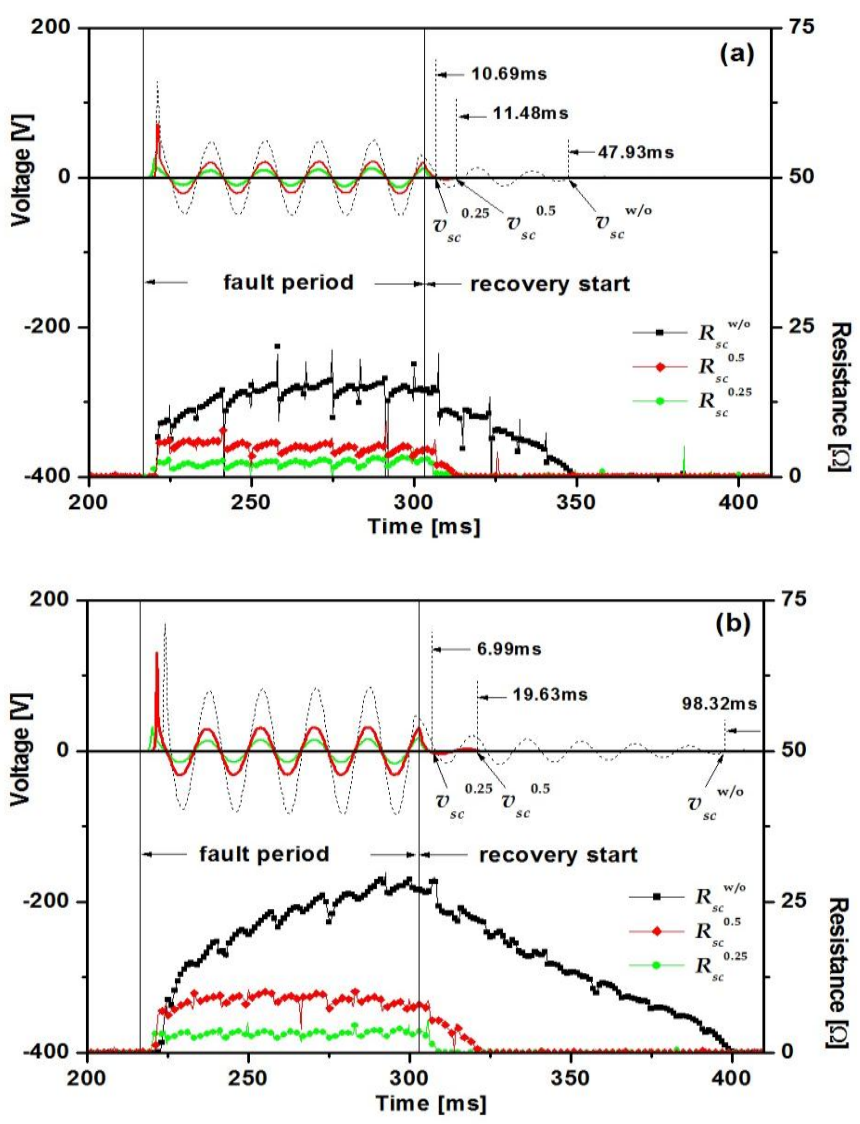

Figure 4. The existence and absence of an isolated transformer and recovery characteristics of the flux-lock type SFCL according to secondary winding change; (a) The additive polarity winding and (b) The subtractive polarity winding

Figure 4 shows the recovery waveforms of the flux-lock type SFCL. The recovery time is the time after finishing the fault until the recovery when the resistance of the element is zero. The recovery time is closely related to the heat energy of the superconducting element, $\mathrm{H}=0.24 \mathrm{I}^{2} \mathrm{RT}$ [cal]. In the voltage waveforms and the resistance curves of Figure 4, 'w/o' expresses the flux-lock type SFCL without the isolated transformer and $n=0.25$ and $n=0.5$ represent the cases in which the winding ratios of the isolated transformer comprising the flux-lock type SFCL are 0.25 and 0.5, respectively.

Figure 4(a) shows the case of the additive polarity winding. With no isolated transformer, the recovery time is 47.93 [ms]. When the winding ratio is $n=0.5$, it is $11.48[\mathrm{~ms}]$, and when the winding ratio is $n=0.25$, it is 10.69 [ms]. Figure $4(\mathrm{~b})$ shows the case of the subtractive polarity winding. With no isolated transformer, the recovery time is 98.32 [ms]. When the winding ratio is $n=0.5$, it is 19.63 [ms], and when the winding ratio is $\mathrm{n}=0.25$, it is 6.99 [ms]. The reason for different recovery times is that the winding direction differs from heat energy generated by the phase transformation of an element. The recovery time of the subtractive polarity winding is longer than that of the additive polarity winding.
Figure 5 presents the bus-voltage sag characteristics of the fluxlock type SFCL. Figure 5(a) shows the peak characteristics of the voltage compensation of a normal line with the additive polarity winding. With no isolated transformer, the initial voltage of the fault occurrence largely increased. Before the fault, at $81[\mathrm{~V}]$, the voltage significantly increased in the beginning, and after two cycles the voltage decreased at 76 [V]. During the fault cycle, the voltage was constant, compensating for 94 [\%] of the voltage before the fault. The bus-voltage sag characteristics according to the secondary winding of the isolated transformer change from 0.25 to 0.5 were similar to $74 \mathrm{~V}$, compensating for $90[\%]$ of the voltage before the fault.

Figure 5(b) shows the peak characteristics of the voltage compensation of a normal line with subtractive polarity winding. With no isolated transformer, the initial voltage of the fault occurrence largely increased at $81[\mathrm{~V}]$, and after two cycles the voltage decreased at 74 [V], compensating for 93 [\%] of the voltage before the fault. The bus-voltage sag characteristics according to the secondary winding change from 0.25 to 0.5 were similar to $69 \mathrm{~V}$, compensating for 85 [\%] of the initial voltage. The bus-voltage sag suppressing characteristics of the additive polarity winding were better than with the subtractive polarity winding. When there was no isolated transformer, the bus-voltage sag suppressing operation showed excellent characteristics.
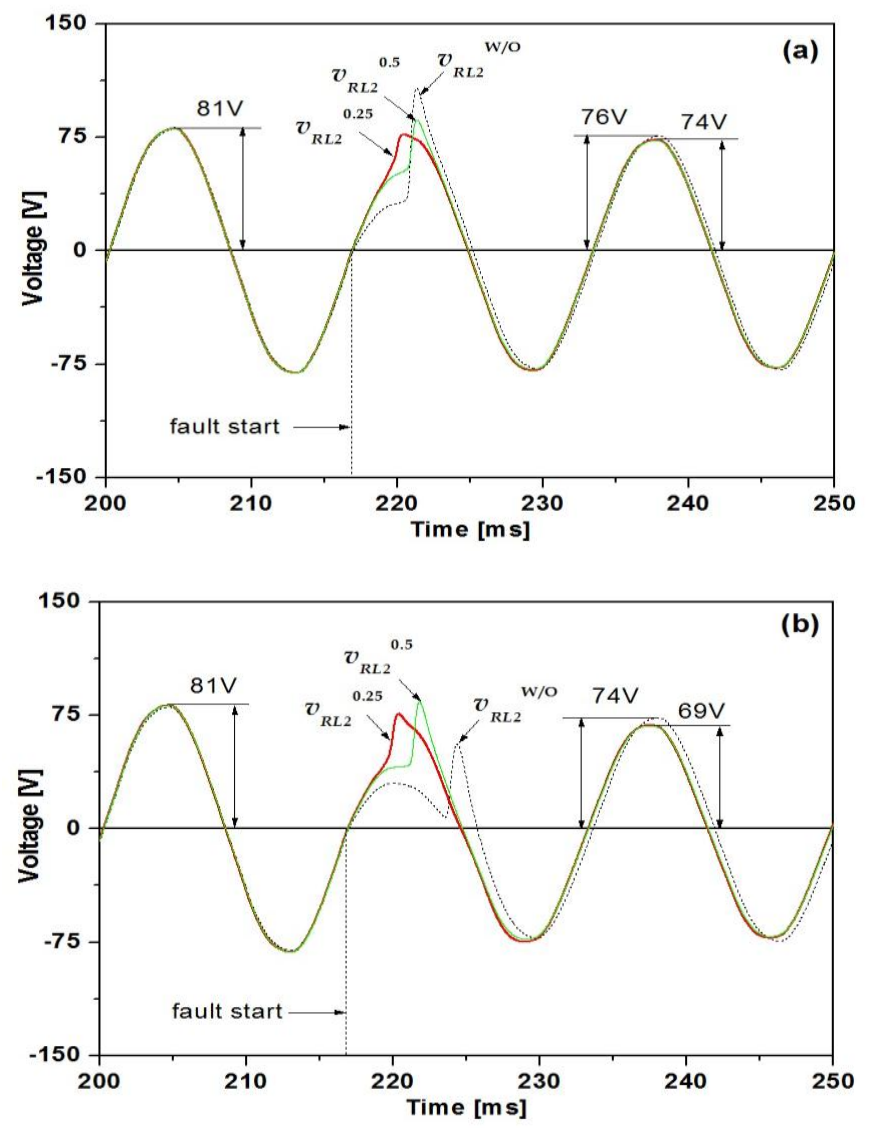

Figure 5. The existence and absence of an isolated transformer and the bus-voltage sag characteristics of the flux-lock type SFCL according to a secondary winding change; (a) The additive polarity winding and (b) The subtractive winding polarity 
Figure 6 shows when a fault occurs, the existence and nonexistence of an isolation transformer, and the relation of the fault current of the flux-lock type SFCL according to the secondary winding change. In Figure $6, i_{\mathrm{FCL}}{ }^{0.25}$ and $i_{\mathrm{FCL}}{ }^{0.5}$ are the fault currents when the winding ratios of the isolated transformer are 0.25 and 0.5 , respectively. $i_{\mathrm{FCL}}{ }^{\text {w/o }}$ is the fault current when the flux-lock type SFCL without the isolated transformer is applied.

Figure 6(a) shows the case of additive polarity winding. When there is no isolation transformer, the fault occurs at the same time as the fault current increases rapidly. It is limited to $1 / 2$ a cycle. When there was no isolation transformer, the first current peak was highest at 31.7[A]. With an isolation transformer, as the winding ratio $\left(n=N_{2 T} / N_{1 T}\right)$ increases from $n=0.25$ to $n=0.5$, the first current peak increases. In addition, after 2 fault cycles, the fault current has similar current peak characteristics with or without an isolation transformer. Figure 6(b) shows the case of subtractive polarity winding. With no isolation transformer, the first current peak is highest at 42.7[A]. After 1 cycle, when the winding ratio is $n=0.25$, the current peak is highest, and at $\mathrm{n}=0.5$, it is low. In the analysis, in the case of using an isolation transformer, the first current peak value decreases. As the secondary coil winding ratio of the isolated transformer is adjusted, the first current peak value can be designed to reduce the burden of superconducting devices.
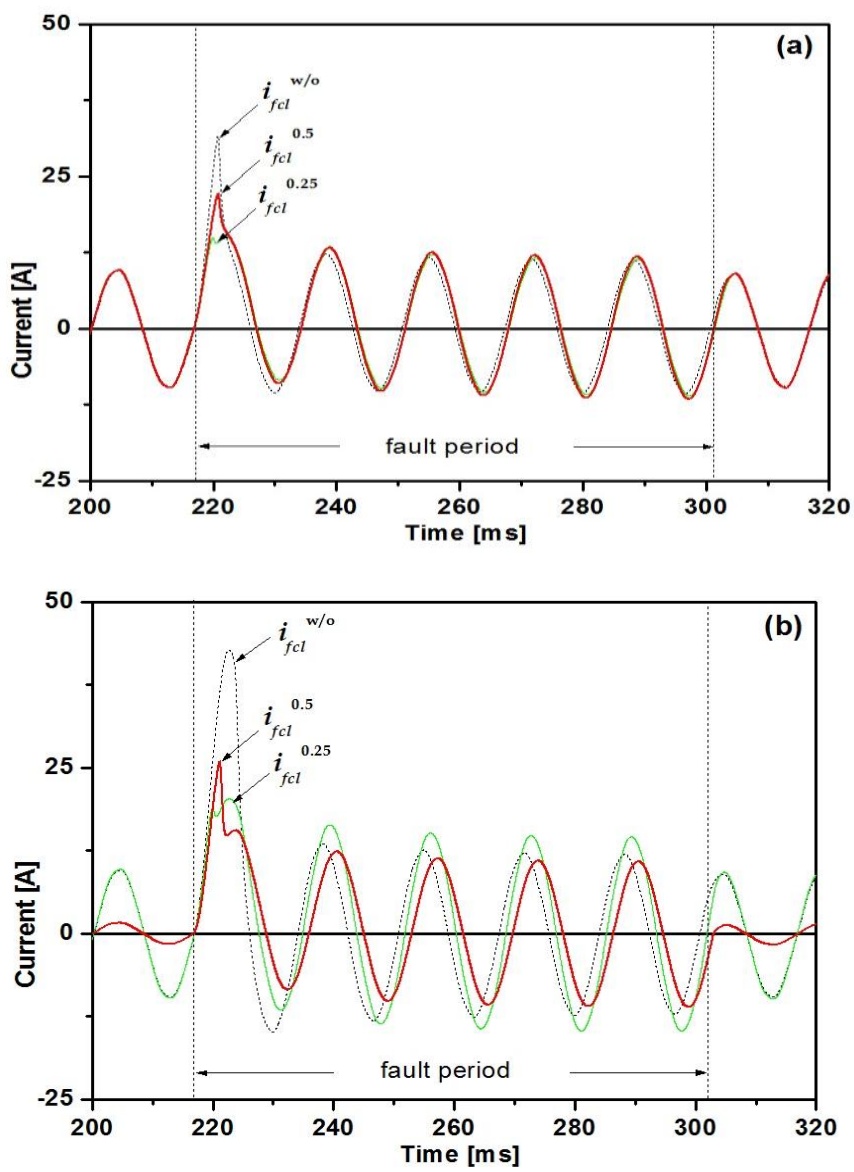

Figure 6. The existence and absence of an isolated transformer and the fault current characteristics of flux-lock type SFCL according to a secondary winding change; (a) The additive polarity winding and (b) The subtractive polarity winding
Figure 7 shows the consumption power of the flux-lock type SFCL. After the fault, the consumption power-burdened superconducting element is high from the first cycle.

The consumption power of this element is $\mathrm{W}=\mathrm{VSC} \cdot \mathrm{isc} \cdot \mathrm{t}$ and is closely related to heat energy $\mathrm{H}=0.24 \mathrm{i} 2 \mathrm{RT}$ [cal]. In Figure 7 , Psc0.25 and Psc0.5 indicate the powers of the superconducting element when the winding ratios of the isolated transformer are 0.25 and 0.5 , respectively. Pscw/o is the power of the superconducting element in the flux-lock type SFCL without the isolated transformer.
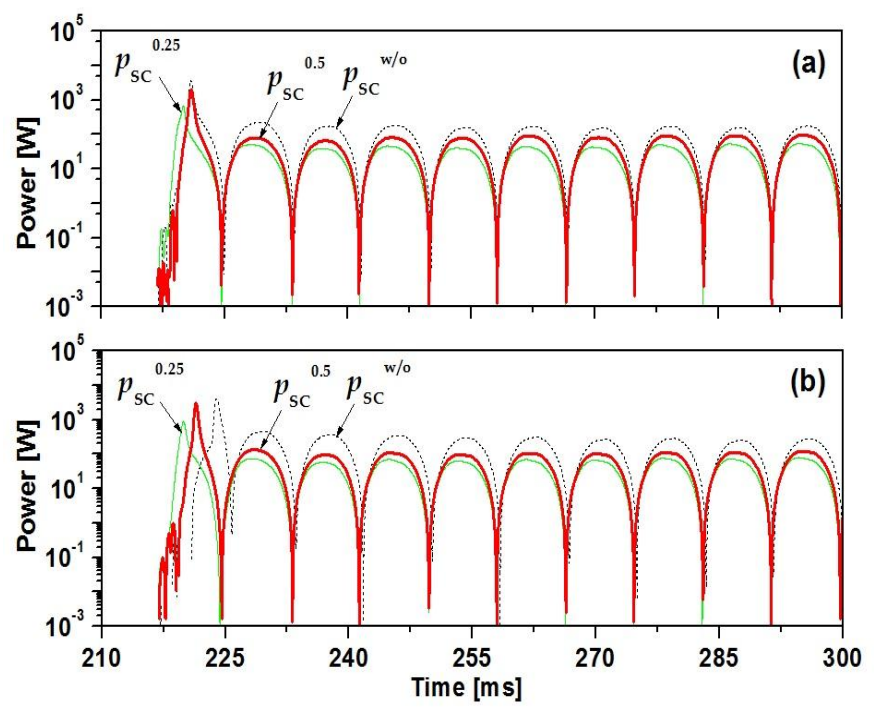

Figure 7. The existence and absence of an isolated transformer and the waveform of consumption power of the flux-lock type SFCL according to a secondary winding change; (a) The additive polarity winding and (b) The subtractive polarity winding

Figure 7(a) gives the case of additive polarity winding. In the case of no isolated transformer, the consumption power is 9,126 [W]. When the secondary coil winding ratio is $n=0.25$, it is 2,551 [W], and when the winding ratio is $n=0.5$, it is 4,579 [W]. Figure 7(b) shows the case of subtractive polarity winding. When there is no isolated transformer, the consumption power is high at 14,828 [W]. When the secondary coil winding ratio is $n=0.25$, it is 3,622 [W], and when the winding ratio is $n=0.5$, it is 6,100 [W]. The consumption power of the superconducting element is high when there is no isolated transformer. When the secondary winding ratio of an isolated transformer increases, the consumption power increases.

\section{CONCLUSION}

In this paper, we examined the flux-lock type SFCL with an isolated transformer, the change of the secondary coil winding ratio of an isolated transformer, and the limiting current according to the existence or absence an isolated transformer. We measured the change in voltage and current to analyze recovery and voltage compensation characteristics. The quench time of the elements had better properties in additive polarity winding than in subtractive. As the winding ratio of the isolated 
transformer increased, the quench time increased. The busvoltage sag suppressing operation possessed excellent properties when there was no isolated transformer. The recovery characteristics are closely related to the consumption energy of the superconducting element. The consumption energy of the superconducting element is determined by the ratio of the secondary winding. The quench and recovery characteristics as a winding method of the isolated transformer can be determined by the secondary coil's winding ratio.

\section{REPERENCES}

[1] E. Thuries, V.D. Pham, Y.Laumond, A. Fevrier, M. Collet, and M. Dekhled, Towards the superconducting fault current limiter, IEEE Trans.on Power Delivery, 6(2), 1991, 801-808.

[2] W. Paul and M. Chen, Superconducting control for surge currents, IEEE Spectrum, 35(5), 1998, 49-54.

[3] L. Salasoo, A. F. Imece, R. W. Delmerico, and R. D. Wyatt, Comparison of superconducting fault limiter concepts in electric utility applications, IEEE Trans. on Appl. Supercond., 5(2), 1995, 1079-1082.

[4] Lin. Ye, and Archie Campbell, Behavior Investigations of Superconducting Fault Current Limiters in Power Systems, IEEE Trans. on Appl. Supercond., 16(2), 2006, 662-665.

[5] C. Lee, H. Kang, M. C. Ahn, T. K. Ko, and B.-Y. Seok, Short-circuit test of a novel solenoid type highTc superconducting fault current limiter, Cryogenics, 47(7-8), 2007, 380-386.

[6] M. Noe and B. R. Oswald, Technical and economical benefits of superconducting fault current limiters in power systems, IEEE Trans. on Appl. Supercond., 9(2), 1999, 1347-1350.

[7] L. Ye and K.-P. Juengst, Modeling and Simulation of High Temperature Resistive Superconducting Fault Current Limiters, IEEE Trans. Appl. Supercond. 14(2), 2004, 839-842.

[8] H. R. Kim, S. W. Yim, S. Y. Oh, and O. B. Hyun, Recovery in Superconducting Fault Current Limiters at Low Applied Voltages, IEEE Trans. Appl. Supercond. 18(2), 2008, 656-659.

[9] K. Shimohata, S. Yokoyama, T. Inaguchi, S. Nakamura and Y. Ozawa, Design of a large current-type fault current limiter with YBCO films, Physica C, 372-376, 2002, 1643-1648.

[10] T. Hoshino, K. M. Salim and M. Nishikawa, Proposal of saturated DC reactor type superconducting fault limiter (SFCL), Cryogenics, 41, 2001, 469-474.

[11] Z. Yang, J. Li, S. Yan. L. Zhang, B. Yu. Li Ren and Y. Liao, Voltage Distribution Characteristic of a FluxCoupling SFCL in Different Operating Conditions, IEEE Trans. on Appl. Supercond., 27(4), 2017, 5601206.

[12] T. H. Han, S. C. Ko and S. H. Lim, Peak Current
Limiting Characteristics of Transformer type SFCL with two non-isolated secondary windings, IEEE Trans. on Appl. Supercond., 28(4), 2018, 5603205.

[13] J. Yuan, Y. Zhong, S. Liao, Y. Gao, K. Murmatsu, J. Jia and B. Chen, Development and Analysis of Bridge-Type saturated-core fault current limiter, IEEE Trans. On Magnetics, 53(11), 2017, 8401005.

[14] Bin Li, Changqi Wang, Wei Hong, Song Yang and Ying Xin, Modeling of the DC Inductive Superconducting Fault Current Limiter, IEEE Trans. on Appl. Supercond., 30(4), 2020, 5601105.

[15] S. H. Lim, H. S. Choi, D. C. Chung, S. C. Ko and B. S. Han, Impedance variation of a flux-lock type SFCL dependent on winding direction between coil 1 and coil 2, IEEE Trans. on Appl. Supercond., 15(2), 2005. 20392042

[16] S. C. Ko, S. H. Lim and T. H. Han, Analysis on fault current limiting and recovery characteristics of a fluxlock type SFCL with an isolated transformer, Physica C, 484, 2013, 263-266.

[17] S. H. Lim, I. K. You and J. C. Kim, Study on Peak Current Limiting Characteristics of a Flux-Lock Type SFCL Using Its Third Winding, IEEE Trans. on Appl. Supercond.,21(3), 2011, 1275-1279. 